\title{
Experimental and numerical investigations on resonant characteristics of a single-layer piezoceramic plate and a cross-ply piezolaminated composite plate
}

\author{
Chien-Ching $\mathrm{Ma}^{\mathrm{a})}$ \\ Department of Mechanical Engineering, National Taiwan University, Taipei, Taiwan 106, \\ Republic of China \\ Hsien-Yang Lin $^{\text {b) }}$ \\ Department of Mechanical Engineering, De Lin Institute of Technology, Tu-Cheng, Taiwan 236, \\ Republic of China \\ Yu-Chih Lin \\ Department of Biomedical Engineering, Yuanpei Institute of Science and Technology Hsinchu, Taiwan 300, \\ Republic of China \\ Yu-Hsi Huang \\ Department of Mechanical Engineering, National Taiwan University, Taipei, Taiwan 106, Republic of China
}

(Received 10 June 2004; revised 19 October 2005; accepted 23 November 2005)

\begin{abstract}
Piezolaminated composite plates are widely used in many industrial applications such as intelligent structures and advanced aerospace structural applications. To improve the dynamic performance of the piezolaminated composite structures, it is necessary to experimentally investigate the resonant characteristics of these structures. Three measurement techniques are used in this study to investigate the vibration behavior of tested specimens. The first method, AF-ESPI (amplitude-fluctuation electronic speckle pattern interferometry), is the major technique for measuring the resonant characteristics of a single-layer piezoceramic plate and a multilayer cross-ply glass fiber reinforced plastics piezolaminated composite plate for completely free conditions. The completely free condition is approximated in experiment by placing and partially sticking the specimen on the surface of a very soft sponge. A thin single-layer piezoceramic plate is first investigated and up to twenty-six transverse modes are presented. Excellent quality of interferometric fringe patterns for vibration mode shapes is presented. The influence of the actual boundaries on the resonant characteristics of the thin piezoceramic plate is discussed. The second method, laser Doppler vibrometer, and the third method, impedance analyzer, are both employed to verify the AF-ESPI results for the piezolaminated composite plate. Both in-plane and out-of-plane resonant frequencies and vibration mode shapes of the piezolaminated composite plate are demonstrated. Finally, numerical computations based on the finite element analysis are presented for comparison with the experimental results. Excellent agreement between the experimental measured data and the numerical calculated results are found for resonant frequencies and mode shapes of the single-layer piezoceramic plate and the cross-ply piezolaminated composite plate. (C) 2006 Acoustical Society of America. [DOI: 10.1121/1.2161428]
\end{abstract}

PACS number(s): 43.40.At, 43.40.Dx [CBB]

Pages: $1476-1486$

\section{INTRODUCTION}

Piezoelectric materials create electric charge when mechanically stressed, conversely, a change in dimensions will be induced upon application of the electric field. Thus, the piezoelectric effect that expresses the connection of electrical and mechanical fields has the built-in performance of the sensing and actuating capacities simultaneously. Over the past few decades, there has been considerable interest in the use and applications of piezoelectric materials in electromechanical transducers, underwater acoustic, electronic packag-

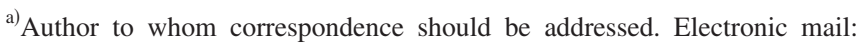
ccma@ntu.edu.tw

${ }^{b)}$ Electronic mail: sylin@dlit.edu.tw
}

ing, resonators, frequency-band filter, vibration damping, and smart structures. One class of piezoelectric materials is the lead zirconate-titanate (PZT) based ceramics. The use of PZT in piezolaminated composite structures has received a great deal of attention because the sensing and actuating capacities become part of the structures in piezolaminated composites, and the ease of integrating piezoceramic materials by means of embedding those to laminated structures. These advantages enhance the growing interest in fast applications of piezolaminated composite structures in the areas of vibration actuation, control, and load/health monitoring. Generally, the piezolaminated composite structures are fabricated by incorporated PZT or polyvinylidene difluoride (PVDF) into piezolaminated composites with fiberreinforced layers. By combining many distinct layers, the 
advantages of each ply can be developed more effectively. Hence, the study of embedded piezoelectric materials in composites has received considerable attention in recent years. However, several researchers have carried out the development of mathematical models and associated computational models to predict the response of piezolaminated composites. Ha et al. ${ }^{1}$ used an eight-node composite brick element and variational principle to construct the FEM formulation for modeling the dynamic and static response of laminated composites containing distributed piezoceramics subjected to both mechanical and electrical loading. Batra and Liang ${ }^{2}$ used the three-dimensional linear theory of elasticity to study the vibrations of simply supported rectangular laminated plate with embedded PZT layers, numerical results for a thin and a thick plate with one embedded actuator layer and one embedded sensor are presented. Abramovich ${ }^{3}$ presented an exact elasticity solution for the forced induced vibrations of a piezolaminated elastic beam. This analysis was carried out by using the method of Fourier series and the solution was exact within the assumptions of linear elasticity and plane strain deformation. Loja et al. ${ }^{4}$ used a family of higher order B-spline finite strip models for the static and free vibration analysis of laminated plates with arbitrary shapes and lay-ups, loading and boundary conditions. The above-mentioned references were usually presented by the theoretical analysis or the numerical computation. Few results used experimental technique to study the vibration characteristics of piezolaminated composite plates. Moreover, the results obtained by experimental methods are always confined in the first few modes. The lack of experimental investigation combined with numerical analysis of resonant characteristics for single-layer piezoceramic plate and the piezolaminated composite plate with an embedded PZT layer in cross-ply fiber reinforced composites are the direct motivation for the study presented herein. For the case of the piezolaminated composite plate, the resonant characteristics of in-plane and out-of-plane motions are investigated. Two noncontact and real-time laser interferometer optical methods, the full-field electronic speckle pattern interferometry (ESPI) technique and the pointwise laser Doppler vibrometer (LDV) technique, are used in this study.

ESPI, which utilizes the information of speckle phenomenon on an optically rough surface, is a full-field and noncontact laser-based optical metrology technique for real-time measurement of surface displacement. Because ESPI excels in the flexibility, real-time, and full-field measurement, it becomes an exciting and highly active field in optical metrology. Furthermore, the image data are digitized by a video camera and processed by a digital signal processing system for the ESPI method, which eliminates the slow and cumbersome process of film development used for conventional technique. The comparative advantage of operation allows ESPI to extend its applications to many practical engineering fields compared with other optical measurement techniques. The application of ESPI to the analysis of vibration study can be traced back to the work by Butters and Leendertz during the 1970s. The detailed description of ESPI techniques can be found in the book written by Jones. ${ }^{6}$ Now, ESPI is a well-established technique for vibration analysis due to ESPI using a video recording and display in real time. The most convenient and widely used experimental setup of vibration measurement by ESPI is the time-averaging method that yields a video image of vibrating object with correlation fringes superimposed. The fringe patterns can be expressed by the zero-order Bessel function and correspond to the contour of constant vibration amplitude or surface displacement. However, the main restrictions of the timeaveraging method are poor image quality, decreased visibility with vibration amplitude, and the lack of the information on vibration phase. Numerous research further developing on the ESPI method has been addressed to overcome these limitations and to extend its usage in practical applications. Hogmoen and Løkberg ${ }^{7}$ proposed a method that was based on sinusoidal phase modulation of reference wave in timeaveraging ESPI for providing information of the vibration phase. Nakadate ${ }^{8}$ used four phase-shifted speckle interferograms for extraction of numerical data of vibration amplitude. Valera et al. ${ }^{9}$ described a stroboscopically illuminated ESPI system by modulating both the illuminating pulse phase relative to the vibration and the optical phase to the interferometer reference beam to generate eight video frames which were processed to generate the vibration phase. Wong ${ }^{10}$ improved the fringe contrast and sensitivity by subtracting two Bessel fringe patterns at two different force levels. Wang et al. ${ }^{11}$ proposed the amplitude-fluctuation ESPI (AF-ESPI) technique that was based on video-signalsubtraction, but the reference image was taken from a vibrating state instead of a free state. The fringe patterns produced by AF-ESPI method have enhanced visibility and reduced noise. Wang et al. ${ }^{12}$ further employed the modal testing technique and the AF-ESPI technique to measure the mode shapes and resonant frequencies of the shadow mask in a multimedia monitor. $\mathrm{Ma}$ and co-worker ${ }^{13}$ further developed the AF-ESPI based on the work of Huang and $\mathrm{Ma}^{14}$ and impedance analyzer for vibrations analysis of piezoelectric materials.

The aim of this work is to investigate experimentally the resonant characteristics of a thin single-layer piezoceramic plate and a piezolaminated composite plate with an embedded piezoceramic layer in cross-ply glass fiber reinforced composites for the completely free boundary condition. The experimental techniques used in this study including the AFESPI, LDV, and impedance analyzer. From the AF-ESPI method, the resonant frequencies and mode shapes of a single-layer piezoceramic plate and a piezolaminated composite plate with free boundary condition are obtained. Up to 26 transverse modes for the thin single-layer piezoceramic plate are presented. The quantitative magnitudes of the fullfield displacements are also indicated in the experimental results for each mode, and the sensitivity is submicrometer. The resonant mode shapes of in-plane and out-of-plane vibrations of the piezolaminated composite plate are also demonstrated. To verify the AF-ESPI results, the LDV, which is a pointwise displacement measurement technique, is also used as the second experimental technique. The third experimental technique, the impedance analyzer, is employed to determine resonant frequencies of in-plane vibration for the piezolami- 


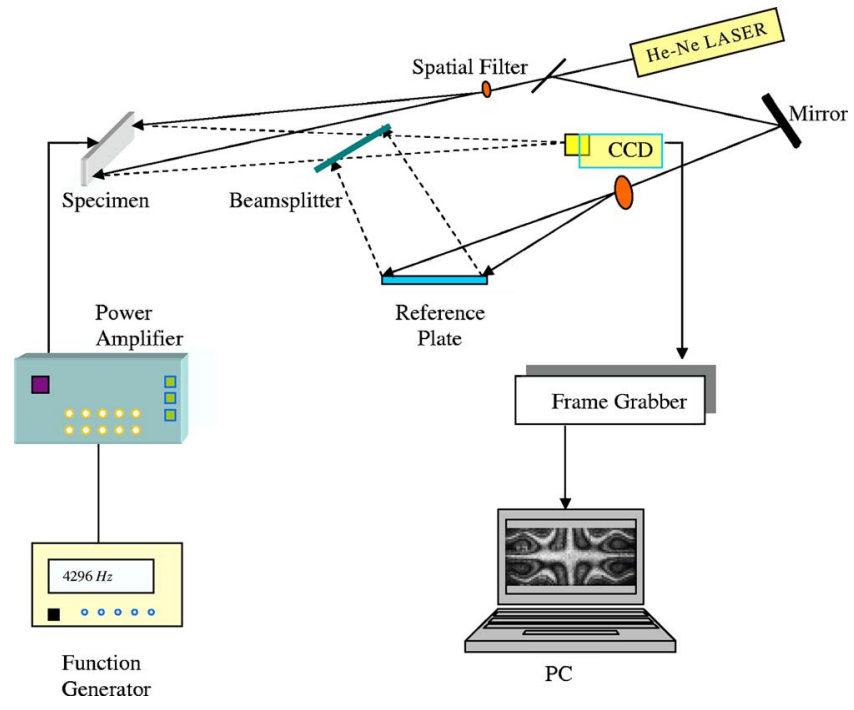

FIG. 1. (Color online) Schematic diagram of AF-ESPI for out-of-plane measurement.

nated composite plate. Furthermore, in light of the results presented in this work, numerical computation based on the finite element method (FEM) utilizing ABAQUS commercial software package ${ }^{15}$ are also made. In practical testing condition, the free boundary in experiment is approximated by placing and partially sticking the specimen on the surface of a very soft sponge. A detailed calculation is made to evaluate the effect of sponge on the vibration behavior of the thin single-layer piezoceramic plate. The excellent agreement of the numerical results in resonant frequencies and mode shapes with experimental measurements is found for the single-layer piezoceramic plate and the cross-ply piezolaminated composite plate.

\section{THEORY OF AF-ESPI METHOD FOR OUT-OF- PLANE AND IN-PLANE MEASUREMENTS}

The time-averaging method is the most commonly used technique of ESPI for experimental vibration measurement. The name "time-averaging" denotes that the vibration measurement includes many periods of object motions during the camera frame period. Two different optical setups (out-ofplane sensitivity and in-plane sensitivity) are employed in this study for the vibration measurement. The optical setups for out-of-plane and in-plane vibration measurements by AFESPI are shown schematically in Figs. 1 and 2, respectively. Fundamentals with regards to the AF-ESPI method for inplane and out-of-plane measurements can be found in Refs. 13 and 14.

\section{A. Out-of-plane vibration}

When the specimen vibrates periodically, the light intensity of the vibrating image detected by a charge-coupled device $(\mathrm{CCD})$ camera can be expressed by the time-averaging method as

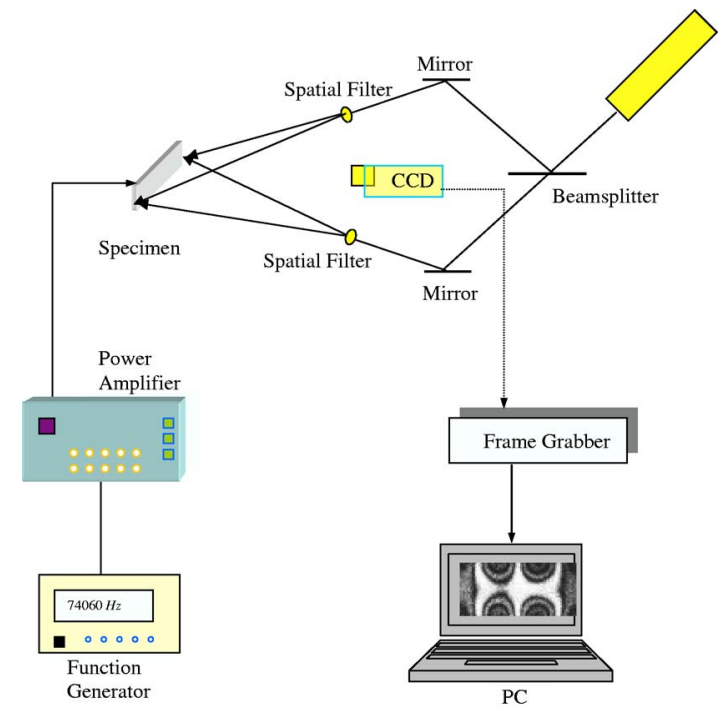

FIG. 2. (Color online) Schematic diagram of AF-ESPI for in-plane measurement.

$$
\begin{aligned}
I= & \frac{1}{\tau} \int_{0}^{\tau}\left\{I_{O}+I_{R}+2 \sqrt{I_{O} I_{R}} \cos \left[\phi+\frac{2 \pi}{\lambda} A(1\right.\right. \\
& +\cos \theta) \cos \omega t]\} d t,
\end{aligned}
$$

where $I_{O}$ is the object light intensity, $I_{R}$ is the reference light intensity, $\phi$ is the random phase difference between the object field and the reference field, $\lambda$ is the wavelength of laser, $A$ is the vibration amplitude, $\theta$ is the angle between the object light and the observation direction, $\tau$ is the CCD refresh time, and $\omega$ is the angular frequency.

Assume that the vibration period is much shorter than the CCD refresh time (i.e., $\tau \gg 2 \pi / \omega$ ) and let

$$
\Gamma=\frac{2 \pi}{\lambda}(1+\cos \theta),
$$

then, Eq. (1) can be approximated to a simplified form as indicated by Jones and Wyke

$$
I_{1}=I_{O}+I_{R}+2 \sqrt{I_{O} I_{R}}(\cos \phi) J_{0}(\Gamma A),
$$

where $J_{0}$ is a zero-order Bessel function of the first kind. As the vibration of specimen goes on, we take the second image by CCD and assume that the vibration amplitude of the second image has changed from $A$ to $A+\Delta A$ due to the electronic noise or the instability of the apparatus. The light intensity of the second image can be represented as

$I_{2}=\frac{1}{\tau} \int_{0}^{\tau}\left\{I_{O}+I_{R}+2 \sqrt{I_{O} I_{R}} \cos [\phi+\Gamma(A+\Delta A) \cos \omega t]\right\} d t$.

Expanding Eq. (4) by using the Taylor series expansion, keeping the first two terms and neglecting the higher order terms, we get

$$
I_{2}=I_{O}+I_{R}+2 \sqrt{I_{O} I_{R}}(\cos \phi)\left[1-\frac{1}{4} \Gamma^{2}(\Delta A)^{2}\right] J_{0}(\Gamma A) .
$$

When these two images, i.e., $I_{1}$ and $I_{2}$, are subtracted and rectified by the image-processing system, the resulting light intensity can be expressed as 


$$
I=\left|I_{2}-I_{1}\right|=\frac{\sqrt{I_{R} I_{O}}}{2}\left|(\cos \phi) \Gamma^{2}(\Delta A)^{2} J_{0}(\Gamma A)\right| .
$$

\section{B. In-plane vibration}

Similar to the out-of-plane vibration case, the first and second image intensities, i.e., $I_{1}$ and $I_{2}$, for in-plane vibration using the time-averaging method are expressed as

$$
I_{1}=I_{O}+I_{R}+2 \sqrt{I_{O} I_{R}}(\cos \phi) J_{0}\left(\Gamma^{\prime} A^{\prime}\right),
$$

and

$$
I_{2}=I_{O}+I_{R}+2 \sqrt{I_{O} I_{R}}(\cos \phi)\left[1-\frac{1}{4} \Gamma^{\prime 2}\left(\Delta A^{\prime}\right)^{2}\right] J_{0}\left(\Gamma^{\prime} A^{\prime}\right),
$$

where $I_{O}$ is the object light intensity, $I_{R}$ is the reference light intensity, $A^{\prime}$ is the vibration amplitude of in-plane vibration, $\Gamma^{\prime}=4 \pi \sin \theta^{\prime} / \lambda$, and $\theta^{\prime}$ is half of the angle between two illumination lights. Subtracting Eq. (8) from Eq. (7) and rectifying by the image processing system, we can obtain the image intensity as

$$
\left[\begin{array}{c}
T_{1} \\
T_{2} \\
T_{3} \\
T_{4} \\
T_{5} \\
T_{6} \\
D_{1} \\
D_{2} \\
D_{3}
\end{array}\right]=\left[\begin{array}{ccccc}
C_{11}^{E} & C_{12}^{E} & C_{13}^{E} & 0 & 0 \\
C_{12}^{E} & C_{11}^{E} & C_{13}^{E} & 0 & 0 \\
C_{13}^{E} & C_{13}^{E} & C_{33}^{E} & 0 & 0 \\
0 & 0 & 0 & C_{44}^{E} & 0 \\
0 & 0 & 0 & 0 & C_{44}^{E} \\
0 & 0 & 0 & 0 & 0 \\
0 & 0 & 0 & 0 & e_{15} \\
0 & 0 & 0 & e_{15} & 0 \\
e_{31} & e_{31} & e_{33} & 0 & 0
\end{array}\right.
$$

From Eq. (10), it is obvious that the complete description of the material characteristic for lead zirconate titanate (PZT) ceramics requires ten material constants; they are five elastic constants $\left(C_{11}^{E}, C_{12}^{E}, C_{13}^{E}, C_{33}^{E}\right.$, and $\left.C_{44}^{E}\right)$, three piezoelectric constants $\left(e_{15}, e_{31}\right.$, and $\left.e_{15}\right)$, and two dielectric constants $\left(\varepsilon_{11}^{s}\right.$ and $\left.\varepsilon_{33}^{s}\right)$. In the following, the resonant characteristics of a single-layer piezoceramic plate and a multilayer cross-ply piezolaminated composite plate are investigated and discussed in detail.

\section{A. The results of the single-layer piezoceramic plate}

The thin single-layer piezoceramic plate with completely free boundary is analyzed first. The plate has the geometrical dimension of $70 \times 25 \times 0.26 \mathrm{~mm}$ and is made of $\mathrm{Pb}(\mathrm{Zr} \cdot \mathrm{Ti}) \mathrm{O}_{3}$ ceramics, the model number is PIC-151 (Physik Instrumente). The material properties of the thin piezoceramic plate are listed in Table I which are provided by Physik Instrumente. The polarization is parallel to the $x_{3}$ axis, and

$$
I=\left|I_{2}-I_{1}\right|=\frac{\sqrt{I_{R} I_{O}}}{2}\left|(\cos \phi) \Gamma^{\prime 2}\left(\Delta A^{\prime}\right)^{2} J_{0}\left(\Gamma^{\prime} A^{\prime}\right)\right| .
$$

From Eqs. (6) and (9), it is interesting to note that the fringe patterns for both the out-of-plane and in-plane vibration motions obtained by AF-ESPI are modulated by a zero-order Bessel function $J_{0}$. Combining the out-of-plane and the inplane optical setups by the AF-ESPI method, we can construct completely the vibration characteristics of piezoelectric materials, including resonant frequencies and mode shapes at the same time.

\section{EXPERIMENTAL AND NUMERICAL RESULTS}

Because the polarized piezoelectric ceramics have the same symmetry as a hexagonal crystal in class $C_{6 v} \mathrm{~mm}$, it can be modeled as a transversely isotropic material. For a transversely isotropic material referred to a Cartesian coordinate system $x_{1}, x_{2}, x_{3}$ and assuming that $x_{1}-x_{2}$ is the isotropic plane and $x_{3}$ is the poling direction, then the constitutive equation for piezoelectric ceramics can be represented in a matrix form as

$$
\left.\begin{array}{cccc}
0 & 0 & 0 & -e_{31} \\
0 & 0 & 0 & -e_{31} \\
0 & 0 & 0 & -e_{33} \\
0 & 0 & -e_{15} & 0 \\
0 & -e_{15} & 0 & 0 \\
\left(C_{11}^{E}-C_{12}^{E}\right) / 2 & 0 & 0 & 0 \\
0 & \varepsilon_{11}^{s} & 0 & 0 \\
0 & 0 & \varepsilon_{11}^{s} & 0 \\
0 & 0 & 0 & \varepsilon_{33}^{s}
\end{array}\right]\left[\begin{array}{c}
S_{1} \\
S_{2} \\
S_{3} \\
S_{4} \\
S_{5} \\
S_{6} \\
E_{1} \\
E_{2} \\
E_{3}
\end{array}\right] .
$$

two opposite faces of the piezoceramic plate are completely coated with silver electrodes. The piezoceramic plate is excited by the application of an ac voltage across electrodes on the two surfaces and has the completely stress-free boundary conditions. Figure 3 is a schematic diagram of the specimen configuration. A self-arranged time-averaging ESPI system is used to perform the experimental measurements for resonant frequencies and corresponding full-field mode shapes. Since the piezoceramic plate is very thin, it will display large transverse (out-of-plane) motion compared to any possible inplane components, especially in the low resonant frequencies. Hence only the optical setup for the out-of-plane sensitivity is used for the single-layer piezoceramic plate.

As shown in Fig. 1, a He-Ne Laser (Uniphase 1135P) with $30 \mathrm{~mW}$ and wavelength $\lambda=632.8 \mathrm{~nm}$ is used as the coherent light source. The emitting laser beam is split into two parts by a variable beamsplitter. One beam is directed toward the tested piezoceramic plate and then reflects to the CCD camera acting as the object beam. The second one which 
TABLE I. Material constants of PIC-151.

\begin{tabular}{cc}
\hline \hline Quality & PIC-151 \\
\hline$C_{11}^{E}\left(\mathrm{~N} / \mathrm{m}^{2}\right)$ & $10.76 \times 10^{10}$ \\
$C_{12}^{E}$ & $6.313 \times 10^{10}$ \\
$C_{13}^{E}$ & $6.386 \times 10^{10}$ \\
$C_{33}^{E}$ & $10.04 \times 10^{10}$ \\
$C_{44}^{E}$ & $1.962 \times 10^{10}$ \\
$C_{66}^{E}$ & $2.224 \times 10^{10}$ \\
$e_{31}(\mathrm{~N} / \mathrm{Vm})$ & -9.52 \\
$e_{33}$ & 15.14 \\
$e_{15}$ & 11.97 \\
$\rho\left(\mathrm{kg} / \mathrm{m}^{3}\right)$ & 7800 \\
$\epsilon_{11}^{s} / \epsilon_{0}$ & 1111 \\
$\epsilon_{33}^{s} / \epsilon_{0}$ & 925 \\
$\epsilon_{0}$ & $8.85 \times 10^{-12}$ \\
\hline \hline
\end{tabular}

serves as a reference beam is illuminated on the surface of a reference plate and reflects into the CCD camera via the beamsplitter. The object and reference beams are combined into the CCD sensor array through a zoom lens (Nikon Micro-Nikkor $55 \mathrm{~mm}$ ). It is important to note that the optical path and the light intensity of these two beams should remain identical in the experimental setup. A CCD camera (Pulnix TM-7CN) and a frame grabber (Dipix P360F) with a digital signal processor on board are used to record and process the images obtained from interferogram of the object and reference beams. Once the specimen vibrates, the interferogram recorded by the CCD camera is stored in an image buffer as a reference image. Then the next frame is grabbed and is subtracted by the image processing system. The CCD camera converts the intensity distribution of the interference pattern of the object into a corresponding video signal at $30 \mathrm{frames} / \mathrm{s}$. The signal is electronically processed and finally converted into an image on the video monitor. The interpretation of the fringe image is similar to the reading of a displacement contour. To achieve a sinusoidal output, a digitally controlled function generator (Hewlett Packard, HP33120A) connected to a power amplifier (NF Electronic Instruments 4005 type) is employed as an input source, which generates periodical exciting force to the specimen.

The detailed experimental procedure of the AF-ESPI technique is performed as follows. First, a reference image is captured after the specimen vibrates, then the second image is taken sequentially, and the reference image is subtracted by the image processing system. If the vibrating frequency is not the resonant frequency, only randomly distributed speckles are displayed and no fringe patterns will be shown. However, if the vibrating frequency is in the neighborhood of the resonant frequency, stationary distinct fringe patterns will be observed in the monitor. Then, the function generator is cautiously and gradually turned; the number of fringes will increase, and the fringe pattern will become clearer but the position of nodal lines will not change as the resonant frequency is approached. From the aforementioned experimental procedure, the resonant frequencies and the corresponding full-field mode shapes can be determined at the same time by using the AF-ESPI optical system. Because the thickness of the piezoceramic plate is very thin, it is easy to excite the

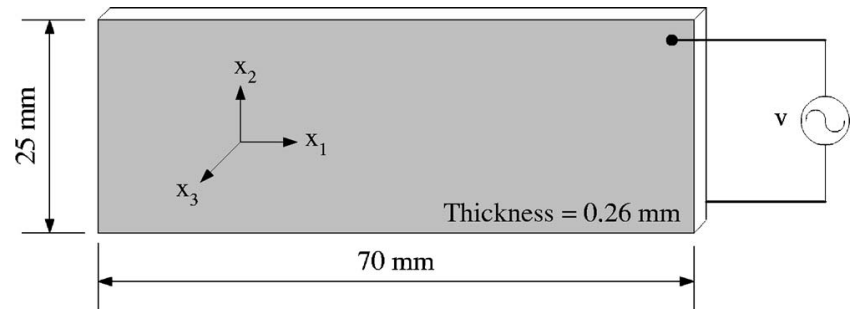

FIG. 3. Schematic diagram for the thin piezoceramic plate.

transverse vibration and up to 26 modes are obtained. The resonant frequencies are ranged from 221 to $5077 \mathrm{~Hz}$.

In addition to the experimental measurement, numerical computations of resonant frequencies as well as mode shapes are also performed by a commercially available software ABAQUS finite element package; a 20-node three-dimensional piezoelectric element (C3D20RE) with reduced integration is used to analyze the problem. Generally, reduced integration provides more accurate results and significantly reduces computational time. Analytically, the boundary condition can be specified as completely free or as any constrained situation. The completely free boundary condition means that the specimen is, in fact, floating in space with no attachment or connection in ground and exhibits rigid body behavior at zero frequency. In testing practice, however, it is almost not realizable and generally not possible to fully achieve this condition. Hence, the specimen must be supported in some manner to approximate the completely free boundary. Since the optical setup as shown in Figs. 1 and 2 is put on a vibration isolated table, the perpendicular direction of the surface of specimen should be in the same plane of the table. It is not possible to hang the specimen in the air by using soft springs. In this study, the completely free boundary is approximated by sticking the single-layer piezoceramic plate onto the surface of a very soft sponge. Since the thickness of the piezoceramic plate is thin, the influence of sponge support on the vibration behavior for the out-of-plane modes is anticipated and should be discussed in detail. Hence, two different boundary conditions are employed in numerical calculations to study the influence of sponge support on the resonant frequencies and mode shapes. The first one is the completely free plate without any constraints or supports except that the bottom and top surfaces of the piezoceramic plate are set to be short-circuited. The second case includes the effect of an elastomeric sponge to model the actual experimental situation. The elastic behavior of highly compressible materials such as sponges or foams is based on the modified form of the strain energy function $U$,

$$
U=\sum_{i=1}^{N} \frac{2 u_{i}}{\alpha_{i}^{2}}\left[\hat{\lambda}_{1}^{\alpha_{i}}+\hat{\lambda}_{2}^{\alpha_{i}}+\hat{\lambda}_{3}^{\alpha_{i}}-3+\frac{1}{\beta_{i}}\left(\left(J^{\mathrm{el}}\right)^{-\alpha_{i} \beta_{i}}-1\right)\right],
$$

where $N$ is the order of strain energy potential, $u_{i}, \alpha_{i}$, and $\beta_{i}$ are material parameters, $\beta_{i}$ is related to the Poisson's ratio by $\beta_{i}=\nu_{i} /\left(1-2 \nu_{i}\right), \hat{\lambda_{i}}=\left(J^{\text {th }}\right)^{-1 / 3} \lambda_{i}$ and $\lambda_{i}$ are the principal stretches. $J^{\text {th }}$ and $J^{\text {el }}$ represent thermo and elastic volume ratio, respectively, and are defined as $J^{\text {th }}=\left(1+\varepsilon^{\text {th }}\right)^{3}, J^{\mathrm{el}}$ $=\lambda_{1} \lambda_{2} \lambda_{3} / J^{\text {th }} ; \varepsilon^{\text {th }}$ is the linear thermal expansion strain that 


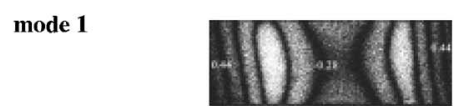

mode 2

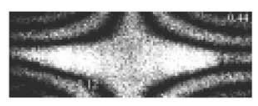

mode 3

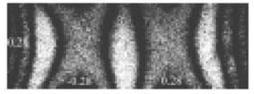

mode 4

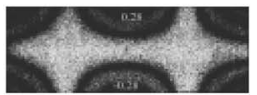

$\operatorname{mode} 5$

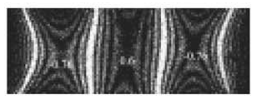

$\operatorname{mode} 6$

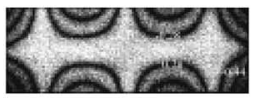

mode 7

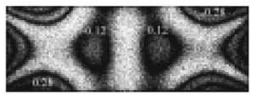

mode 8

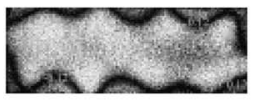

mode 9

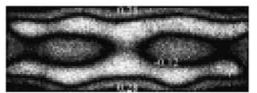

mode 10

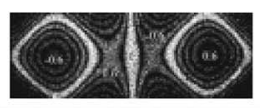

mode 11

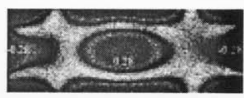

mode 12

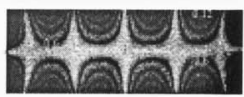

mode 13

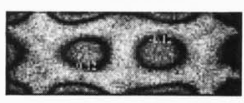

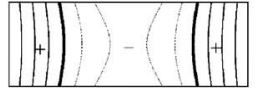
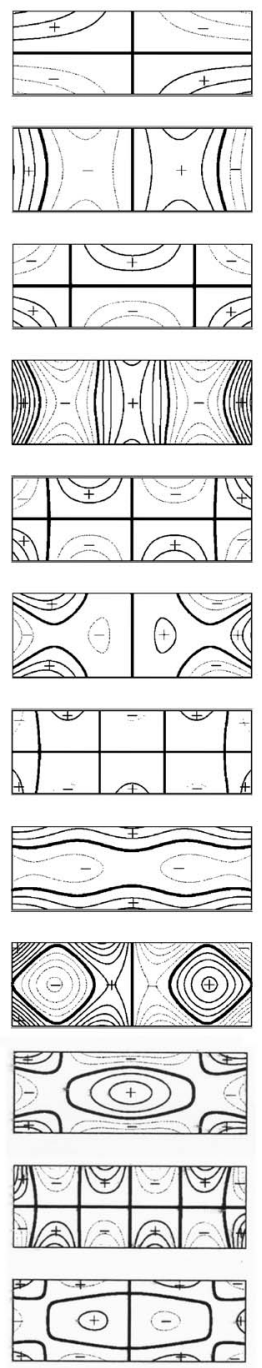

mode 14

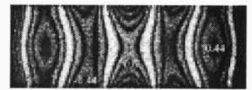

mode 15

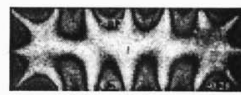

mode 16

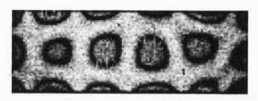

mode 17

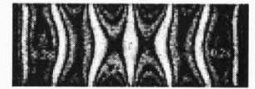

mode 18

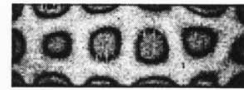

mode 19

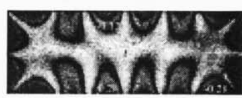

mode 20

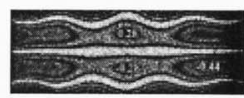

mode 21

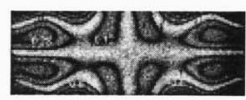

mode 22

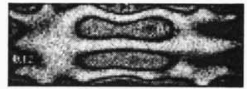

mode 23

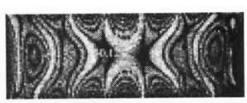

mode 24

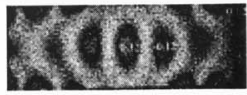

mode 25

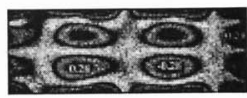

mode 26

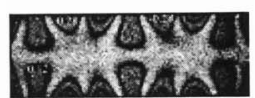

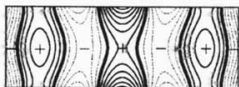
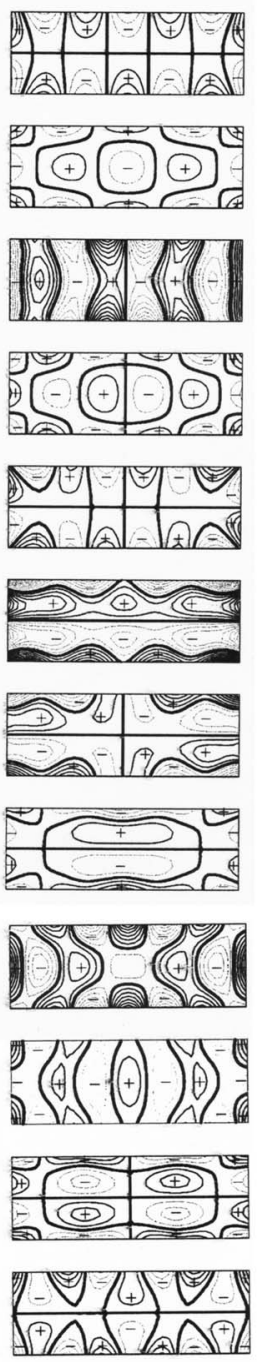

FIG. 4. The out-of-plane mode shapes of the thin piezoceramic plate obtained by AF-ESPI and FEM.

is obtained from the temperature and the isotropic thermal expansion coefficient. In this study, the material parameters of the soft sponge are determined by putting the counterweights on the upper surface of the sponge and measuring the correspondent displacements. The value of $N$ is set to be 1 . The material parameters $u_{i}, \alpha_{i}$, and $\beta_{i}$ are determined to be $10303.2,25.0$, and 0 , respectively, from the measurement of the nominal stress and strain in a simple experiment. Since we do not take the thermal effect into account, hence $\varepsilon^{\text {th }}=0, J^{\text {th }}=1$, and $J^{\mathrm{el}}=\lambda_{1} \lambda_{2} \lambda_{3}$.

Figure 4 presents the detailed mode shapes of the PIC151 single-layer piezoceramic plate obtained by AF-ESPI with out-of-plane sensitivity and FEM. The excellent quality of the interferometric fringe pattern obtained from the AFESPI method is demonstrated. For the finite element results as shown on the right-hand side of Fig. 4, the dashed lines and the "-" sign indicate the concave displacements, while the solid lines and the "+" sign denote convex displacements. The transition from solid lines to dashed lines corre- sponds to a zero displacement line or a nodal line that is represented as a bold black line. The zero-order fringes, which are the brightest fringes on the experimental results, represent the nodal lines of the piezoceramic plate at resonant frequencies. The rest of the fringes are contour of constant displacements. The mode shapes obtained from experimental results can be checked by the nodal lines and fringe patterns with the numerical finite element calculations. The related amplitude $A_{i}, i=1,2,3,4 \ldots, n$ for the $i$ th fringe in the experimental results can be quantitatively calculated by the roots of $J_{0}(\Gamma A)=0$ as indicated in Eq. (6). We use $\theta=10^{\circ}$ for the experimental setup and $\lambda=632.8 \mathrm{~nm}$; the related vibration amplitudes for the first six dark fringes are $A_{i}, i=1-6$, $=0.12,0.28,0.44,0.6,0.76$, and $0.92 \mu \mathrm{m}$. The maximum value of the vibration displacement and related amplitudes of some fringes in the experimental results are also indicated in Fig. 4. Note that the vibration displacements obtained in this study are in the order of submicrometer. Excellent agree- 
TABLE II. Results of resonant frequencies for the out-of-plane vibration obtained from AF-ESPI and FEM for a thin PIC-151 plate (FEM I represents the complete free case, FEM II represents the sponge effect included, Error is compared with AF-ESPI results).

\begin{tabular}{|c|c|c|c|}
\hline Mode & AF-ESPI (Hz) & FEM I (Error \%) & FEM II (Error \%) \\
\hline 1 & 221 & $165.3(-33.73)$ & $220.7(-0.12)$ \\
\hline 2 & 306 & $257.4(-18.90)$ & $319.9(4.35)$ \\
\hline 3 & 525 & $466.9(-11.07)$ & $503.5(-4.28)$ \\
\hline 4 & 613 & $556.6(-10.14)$ & $597.3(-2.62)$ \\
\hline 5 & 953 & $923.5(-3.20)$ & $950.0(-0.31)$ \\
\hline 6 & 990 & $936.8(-5.68)$ & $968.6(-2.21)$ \\
\hline 7 & 1466 & $1419.1(-3.30)$ & $1444.5(-1.49)$ \\
\hline 8 & 1491 & $1432.2(-4.11)$ & $1459.2(-2.18)$ \\
\hline 9 & 1509 & $1503.2(-0.39)$ & $1527.8(1.23)$ \\
\hline 10 & 1746 & $1746.7(0.04)$ & $1769.4(1.32)$ \\
\hline 11 & 1896 & $1859.5(-1.96)$ & $1880.1(-0.85)$ \\
\hline 12 & 2127 & $2066.1(-2.95)$ & $2094.7(-1.54)$ \\
\hline 13 & 2401 & $2345.5(-2.37)$ & $2364.6(-1.54)$ \\
\hline 14 & 2486 & $2471.9(-0.57)$ & $2499.8(0.55)$ \\
\hline 15 & 2905 & $2844.4(-2.13)$ & $2881.9(-0.80)$ \\
\hline 16 & 3022 & $2963.4(-1.98)$ & $2984.8(-1.25)$ \\
\hline 17 & 3422 & $3435.3(0.39)$ & $3481.8(1.72)$ \\
\hline 18 & 3785 & $3705.5(-2.15)$ & $3760.5(-0.65)$ \\
\hline 19 & 3799 & $3723.7(-2.02)$ & $3764.2(-0.92)$ \\
\hline 20 & 4097 & 4104.9(0.19) & $4166.3(1.66)$ \\
\hline 21 & 4296 & $4298.5(0.06)$ & $4365.2(1.59)$ \\
\hline 22 & 4461 & $4489.7(0.64)$ & $4544.5(1.84)$ \\
\hline 23 & 4529 & $4521.0(-0.18)$ & $4588.5(1.30)$ \\
\hline 24 & 4702 & $4687.5(-0.31)$ & $4772.8(1.51)$ \\
\hline 25 & 4985 & $5000.8(0.32)$ & $5074.7(1.77)$ \\
\hline 26 & 5077 & $5088.9(0.23)$ & $5194(2.30)$ \\
\hline
\end{tabular}

ments of the experimental measurement and numerical calculation are found for all the resonant modes.

Table II tabulates the first 26 resonant frequencies obtained by AF-ESPI and FEM, where FEM I represents the first case, the completely free boundary, FEM II represents the second case that includes the effect of the soft sponge. Compare resonant frequencies measured by the AF-ESPI method with the results predicted by FEM I, the larger discrepancy is greater than $10 \%$ and occurs at the first four modes; good agreement is obtained for the higher vibration modes. However, if we compare the resonant frequencies obtained by AF-ESPI and FEM II, excellent agreement is achieved for all the vibration modes and the difference for the first four modes are significantly reduced. It can be concluded at this point that the soft sponge support has a significant influence on the resonant frequencies of the lower resonant modes for thin piezoceramic plates. It is worthy to note that the sequence of all the 26 modes calculated from FEM II is the same as that obtained by the experimental observation. However, the sequence of the 18th and 19th modes are exchanged from the prediction by FEM I. It is indicated from Table II that the finite element simulation underestimates the resonant frequencies if the effect of the sponge support is not included.

It is noted that if the geometrical dimension and material properties are known for the tested specimen and the convergence investigation has been performed, then resonant frequencies can be accurately determined from numerical cal-

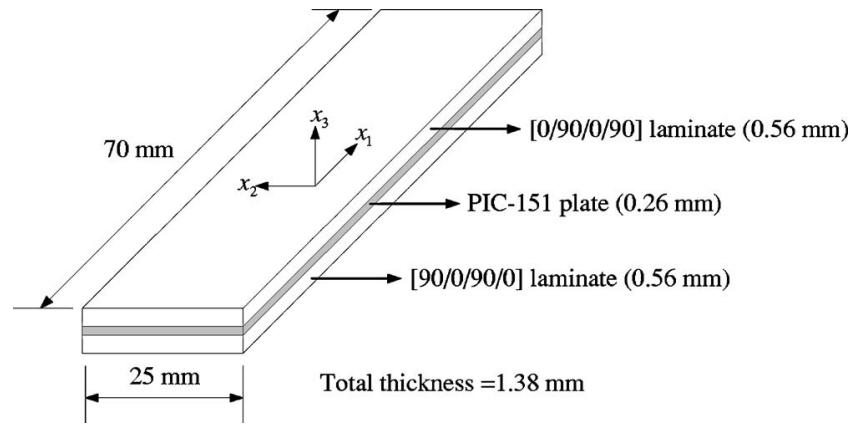

FIG. 5. Geometrical configuration of the GFRP piezolaminated composite plate.

culations. However, some difficulties may arise for the AFESPI experimental measurement of resonant frequencies. From the experimental measurement point of view, it is well known that there is a bandwidth for the resonant frequency determination. Some resonant frequencies have very narrow bandwidth for only several hertz and the resonant frequency can be easily and accurately determined experimentally. However, some resonant frequencies have relatively wide bandwidth for hundreds of hertz. The number of fringes is nearly the same within the bandwidth of resonant frequencies and the experimental result is not easy to accurately determine. Furthermore, the material properties provided by Physik Instrumente and the boundary condition of the specimen might be different between experimental measurement and numerical calculation. The above-mentioned main reasons will result in the discrepancy of resonant frequencies measured by experiment and predicted by finite element calculation.

\section{B. The results of the multilayer cross-ply piezolaminated composite plate}

The resonant characteristics of in-plane and out-of-plane vibrations for a cross-ply piezolaminated composite plate with an embedded piezoceramic layer are investigated. The optical systems for the out-of-plane and the in-plane measurements are indicated in Figs. 1 and 2, respectively. The piezolaminated composite plate has the size of $70 \times 25$ $\times 1.38 \mathrm{~mm}$. Figure 5 shows its schematic illustration of the specimen, a thin piezoceramic plate that has the same size and material property as the first experimental specimen is embedded in the middle of the cross-ply fiberglass reinforced plastic materials. The stacking sequence of the piezolaminated composite plate is [0/90/0/90/PIC-151/90/0/90/0]. The two opposite faces of the thin piezoceramic plate are completely coated with silver electrodes, and this cross-ply piezolaminated composite plate is excited by the application of a time-harmonic voltage across electrodes on the two sur-

TABLE III. Material constants of fiberglass reinforced lamina used in FEM I (unidirectional).

\begin{tabular}{lccccc}
\hline \hline $\begin{array}{c}\text { Material } \\
\text { properties }\end{array}$ & $E_{11}(\mathrm{GPa})$ & $E_{22}(\mathrm{GPa})$ & $\nu_{12}$ & $G_{12}(\mathrm{GPa})$ & $\rho$ \\
\hline Magnitude & 36.11 & 8.8 & 0.32 & 3.48 & $1820\left(\mathrm{~kg} / \mathrm{m}^{3}\right)$ \\
\hline \hline
\end{tabular}


TABLE IV. Material constants of fiberglass reinforced lamina used in FEM II and FEM III (unidirectional).

\begin{tabular}{lcccccccc}
\hline \hline $\begin{array}{c}\text { Material } \\
\text { properties }\end{array}$ & $E_{11}$ & $E_{22}$ & $E_{33}$ & $\nu_{12}$ & $\nu_{13}$ & $\nu_{23}$ & $G_{12}$ & $G_{13}$ \\
\hline Magnitude & $\begin{array}{c}36.11 \\
(\mathrm{GPa})\end{array}$ & $\begin{array}{c}8.8 \\
(\mathrm{GPa})\end{array}$ & $\begin{array}{c}8.8 \\
(\mathrm{GPa})\end{array}$ & 0.32 & 0.32 & 0.43 & $\begin{array}{c}3.48 \\
(\mathrm{GPa})\end{array}$ & $\begin{array}{c}3.48 \\
(\mathrm{GPa})\end{array}$ \\
\hline \hline
\end{tabular}

faces of the embedded thin piezoceramic plate. The fiberglass used is a continuous filament "E" type, the composite prepreg tape is manufactured by the Forthtrack Co., Ltd, and the model number is FT-150G. It consists of fiberglass precoated with the thermosetting resin, the composition of epoxy resin and fiberglass is $37 \%$ and $63 \%$, respectively. The fabrication of these laminated composite structures involves simply laying-up the prepreg at the required orientation on a forming mold, stacking layers of prepreg in the required stacking sequence, and then curing the assembly under elevated temperature and pressure of $130{ }^{\circ} \mathrm{C}$ and $50 \mathrm{psi}$ for $2 \mathrm{~h}$. Table III lists the material properties of the fiberglassreinforced lamina measured by the material testing system (MTS) and strain gauges, where $E_{11}$ and $E_{22}$ are Young's moduli in the longitudinal and transverse directions, respectively; $\nu_{12}$ is the Poisson's ratio, $G_{12}$ is the in-plane shear modulus. The material properties listed in Table III are enough for the two-dimensional FEM calculation. However, more material constants of the fiberglass-reinforced lamina are needed for the complicated three-dimensional numerical calculation. Since the axial axis of the fiber in the fiberglassreinforced composite is along the $x_{1}$ axis, it is often assumed that the material behavior in the $x_{2}$ axis is identical to the material behavior in the $x_{3}$ axis. For this situation, we have $E_{33}=E_{22}=8.8 \mathrm{GPa}, \nu_{13}=\nu_{12}=0.32$, and $G_{13}=G_{12}=3.48 \mathrm{GPa}$. We further assume that the material is isotropic in the $x_{2}$ $-x_{3}$ plane and $\nu_{23}=0.43$. The shear modulus can then be evaluated from $G_{23}=E_{2} / 2\left(1+\nu_{23}\right)=3.1 \mathrm{GPa}$. Hence the other five elastic constants $\left(E_{33}, \nu_{13}, \nu_{23}, G_{13}, G_{23}\right)$ are properly chosen without using the experimental measurement and the complete list of the material properties is indicated in Table IV. Because the piezolaminated composite plate has the thickness of $1.38 \mathrm{~cm}$, both the in-plane and out-of-plane vibration modes will be discussed. The optical setups of AFESPI for the in-plane and out-of-plane sensitivity are used to investigate the resonant characteristics of the piezolaminated composite plate. The vibration analysis of angle-ply laminated composite plates with an embedded piezoceramic layer was investigated by Lin et al. ${ }^{16}$ They analyzed the resonant frequencies and correspondent mode shapes of five different angle-plies for out-of-plane modes only.

In FEM simulation for the multilayer cross-ply piezolaminated composite plate, three formulations based on different models are used to evaluate the resonant vibration characteristics of the piezolaminated plate. The Lanczos eigensolver is used to perform eigenvalue extraction for calculating the natural frequencies and corresponding mode shapes. The numerical results for resonant frequencies are listed in Tables V and VI and are denoted as FEM I, FEM II, and FEM III. FEM I uses the plane shell element (S9R5) with nine-node, five degrees of freedom at the node, and the Kirchhoff constraint imposed numerically is used to model the resonant vibration behaviors of the piezolaminated plate. The material properties used in the numerical calculation for FEM I are indicated in Tables I and III. A local coordinate system for definition of the material properties in each layer is used. FEM II uses the solid element (C3D20R) to model the resonant vibration behavior of the piezolaminated plate. The total numbers of elements and nodes used are 2646 and 13 974, respectively. Because the piezoelectric coupling effect is active only in piezoelectric elements, the effect of the embedded piezoceramic layer on the resonant vibration characteristics is not included in FEM I and FEM II. To investigate the piezoelectric coupling effect of the embedded piezoceramic layer, the solid element (C3D20R) and the threedimensional piezoelectric element (C3D20RE) are used in FEM III to model the behavior of laminated composite layers and the embedded piezoceramic layer, respectively. The elements C3D20R and C3D20RE are 20-node quadratic brick, reduced integration, three-dimensional continuum stress/ displacement and piezoelectric element, respectively. The total numbers of elements and nodes used in FEM III are the

TABLE V. Results of resonant frequencies for the out-of-plane vibration obtained from AF-ESPI, LDV, and FEM for the piezolaminated composite plate. (Error is compared with AF-ESPI values.)

\begin{tabular}{cccccc}
\hline \hline Mode & AF-ESPI (Hz) & LDV (Error \%) & FEM I (Error \%) & FEM II (Error \%) & FEM III (Error \%) \\
\hline 1 & 976 & $980(0.41)$ & $885(-9.32)$ & $806(-17.39)$ & $809(-17.12)$ \\
2 & 1005 & $1030(2.49)$ & $918(-8.66)$ & $889(-11.56)$ & $889(-11.56)$ \\
3 & 2185 & $2240(2.52)$ & $2082(-4.71)$ & $1787(-9.06)$ & $1989(-9.00)$ \\
4 & 2605 & $2600(-0.19)$ & $2440(-6.33)$ & $2215(-14.96)$ & $2223(-14.66)$ \\
5 & 3838 & $3970(3.44)$ & $3744(-2.45)$ & $3508(-8.60)$ & $3514(-8.43)$ \\
6 & 4964 & $5050(1.73)$ & $4778(-3.75)$ & $4316(-13.06)$ & $4332(-12.74)$ \\
7 & 6197 & $6070(-2.05)$ & $5847(-5.65)$ & $5602(-9.60)$ & $5617(-9.37)$ \\
8 & 6362 & $6360(-0.03)$ & $6083(-4.39)$ & $6112(-3.94)$ & $6140(-3.50)$ \\
9 & 6697 & $6600(-1.45)$ & $6113(-8.72)$ & $6327(-5.53)$ & $6350(-5.18)$ \\
10 & 7338 & $7510(2.34)$ & $7028(-4.22)$ & $7117(-3.02)$ & $7149(-2.58)$ \\
\hline \hline
\end{tabular}


TABLE VI. Convergence test of out-of-plane resonant frequencies from FEM III of the piezolaminated composite plate.

\begin{tabular}{|c|c|c|c|c|c|c|c|c|c|c|}
\hline No. of elements & 120 & 210 & 324 & 552 & 702 & 1188 & 1638 & 2112 & 2646 & 3078 \\
\hline Mode 1 & 808.3 & 808.5 & 808.7 & 808.8 & 808.8 & 808.9 & 808.9 & 808.9 & 808.9 & 808.9 \\
\hline Mode 2 & 895.1 & 893.5 & 892.4 & 891.0 & 890.5 & 889.6 & 889.3 & 889.0 & 888.9 & 888.8 \\
\hline Mode 3 & 2000.2 & 1997.2 & 1995.1 & 1992.6 & 1991.8 & 1990.2 & 1989.6 & 1989.1 & 1988.8 & 1988.7 \\
\hline Mode 4 & 2221.9 & 2221.8 & 2222.1 & 2222.5 & 2222.6 & 2222.8 & 2222.9 & 2222.9 & 2223 & 2223 \\
\hline Mode 5 & 3529.3 & 3524.3 & 3521.6 & 3518.7 & 3517.8 & 3516 & 3515.2 & 3514.7 & 3514.3 & 3514.1 \\
\hline Mode 6 & 4336.9 & 4330.8 & 4330.2 & 4330.9 & 4331 & 4331.4 & 4331.6 & 4331.6 & 4331.7 & 4331.7 \\
\hline Mode 7 & 5637.2 & 5626.2 & 5622.8 & 5620.4 & 5619.6 & 5618.1 & 5617.4 & 5616.9 & 5616.6 & 5616.4 \\
\hline Mode 8 & 6249.4 & 6204.4 & 6182.7 & 6162 & 6156.3 & 6146.7 & 6143.3 & 6141.1 & 6139.6 & 6139 \\
\hline Mode 9 & 6454.2 & 6412.6 & 6392.4 & 6372.7 & 6367.1 & 6357.4 & 6353.9 & 6351.6 & 6350 & 6349.4 \\
\hline Mode 10 & 7190.8 & 7156.5 & 7149.8 & 7148.6 & 7148.4 & 7148.5 & 7148.5 & 7148.5 & 7148.6 & 7148.6 \\
\hline
\end{tabular}

same as the case in FEM II. For the case of FEM III, the electrical boundary condition is set to be short-circuited on both the top and bottom surfaces. It is obvious that FEM II and FEM III are more time consuming for the numerical calculation. The material properties used in the numerical calculation for FEM II and FEM III are indicated in Tables I and IV.

Figures 6 and 7 show the out-of-plane and in-plane resonant mode shapes obtained by AF-ESPI and FEM I, respectively. The first ten transverse (out-of-plane) modes of the piezolaminated composite plate are indicated in Fig. 6. It is found that the sequence of the third and the fourth, the fifth and the sixth, the seventh and the ninth, the tenth and the eleventh mode shapes of the piezolaminated composite plate interchanges as compared with the mode shapes of the single-layer piezoceramic plate as shown in Fig. 4. The sequence and mode shapes for out-of plane vibration modes predicted by FEM I, FEM II, and FEM III are almost the same, only the sequence of the seventh and the eighth modes are exchanged for FEM I and FEM II (FEM III). Owing to the geometric configuration of the rectangular piezolaminated composite plate, two in-plane vibration modes for each resonant frequency in the $x_{1}$ and $x_{2}$ directions are displayed in order to present the complete characteristics of the inplane vibration behavior. Figure 7 shows the results of the first six in-plane mode shapes, the $U$ field and the $V$ field denote the full-field vibration displacement along the $x_{1}$ and the $x_{2}$ directions, respectively. The $V$ field of the sixth mode cannot be measured by the AF-ESPI, this is due to the reason that the vibration amplitude for this mode is too small and is beyond the sensitivity of the in-plane setup of the AF-ESPI. It is shown in Fig. 7 that the characteristics of the vibration displacements for the $U$ and $V$ fields are quite different. Excellent agreement of the experimental measurement and numerical calculation is found for both the out-of-plane and in-plane vibration modes as indicated in Figs. 6 and 7.

Because the electrical impedance will drop to a local minimum when it is in resonance, the resonant frequencies can also be obtained by the impedance analysis. Figure 8 shows the impedance curve of this piezolaminated composite plate measured by a HP4194A impedance/gain-phase analyzer. The local minimum appearing in the impedance curve corresponds to the resonant frequencies. Because the summa- tion of the induced charge distributed over the electrode surfaces is zero for out-of-plane modes, only the in-plane modes can be measured by the impedance analysis.

The LDV is a pointwise displacement measurement technique and is sensitive to the out-of-plane displacement. Hence, the LDV is used as the second experimental technique to verify the experimental results of the transverse resonant frequencies for the piezolaminated composite plate obtained by AF-ESPI. The optical system of LDV is based on the principle of Michelson interferometer and the Doppler effect. A built-in dynamic signal analyzer (DSA) is integrated into the LDV system to become the LDV-DSA sys-
AF-ESPI

mode 1

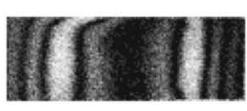

mode 2

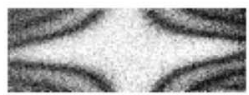

mode 3

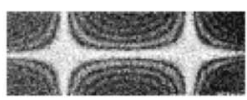

mode 4

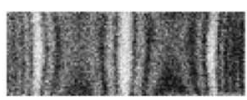

mode 5

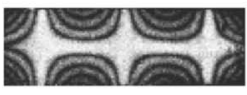

mode 6

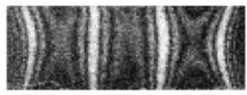

mode 7

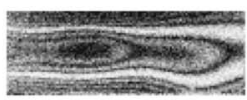

mode 8

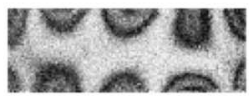

mode 9

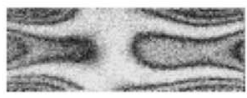

mode 10

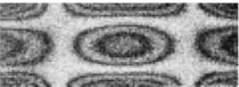

FEM
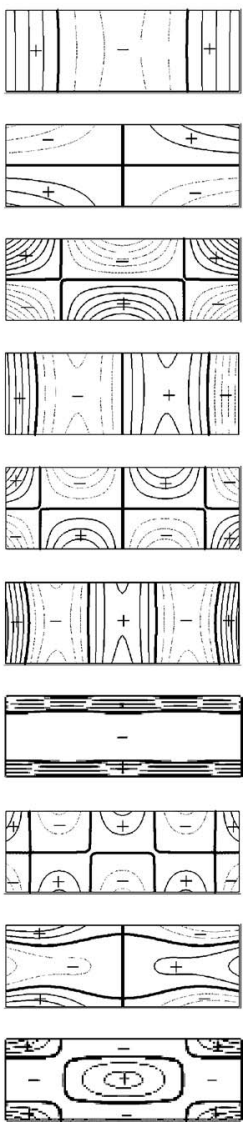

FIG. 6. The out-of-plane mode shapes of the GFRP piezolaminated composite plate obtained by AF-ESPI and FEM. 


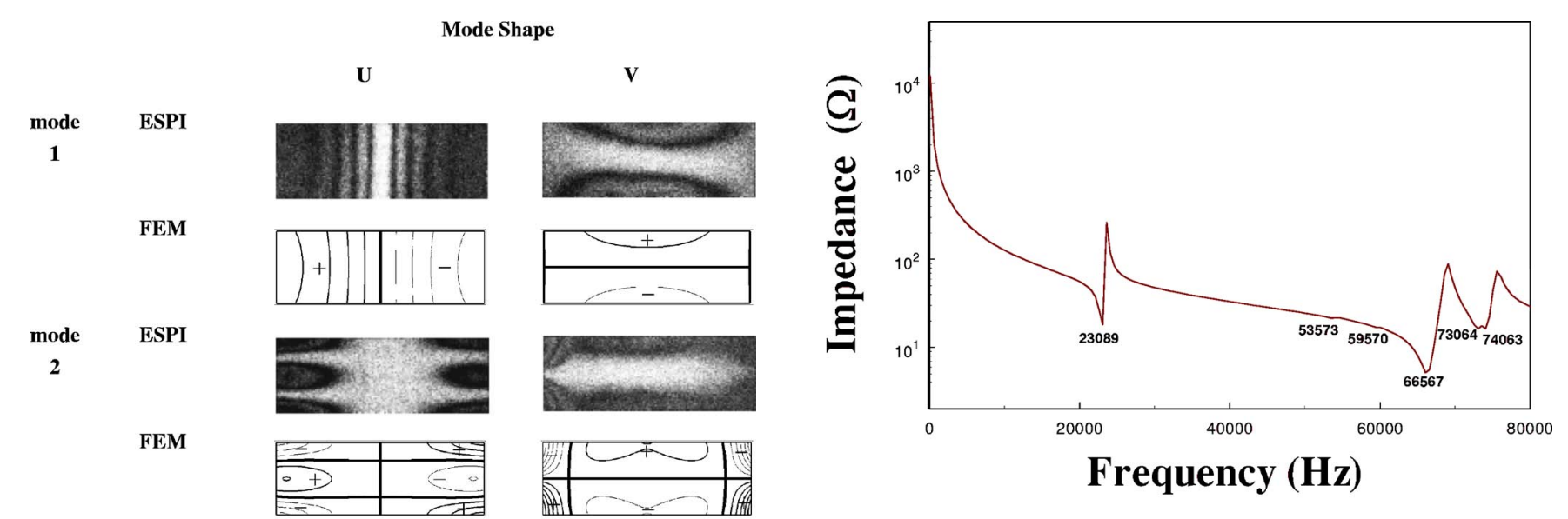

FIG. 8. Impedance curve of the piezolaminated composite plate.

FEM when compared with the AF-ESPI results. We can see that the resonant frequencies measured from these two optical methods agree very well. The resonant frequencies of out-of-plane modes predicted by FEM II and FEM III (threedimensional analysis) are almost the same. The two experimental measured resonant frequencies (AF-ESPI and LDV) are closely related to the predicted results by FEM I (twodimensional analysis), but have larger discrepancies when compared with the results by FEM II and FEM III. This is due to the fact that five of the material properties used for the numerical calculations by FEM II and FEM III are not directly obtained from the experimental measurement. The convergence test of the finite element result FEM III has been performed and the result is indicated in Table VI. It shows that the numerical result of the resonant frequency has no difference as the element number over 2000. It is indicated previously that the total number of element used for FEM II an FEM III is 2646.

Table VII shows the first six resonant frequencies obtained by AF-ESPI, impedance, and FEM for in-plane vibration modes. It is worthy to note that the results obtained by impedance analyzer, AF-ESPI and FEM I are in excellent agreement. The largest discrepancy is only $1.57 \%$. The discrepancy of the results between experimental measurement

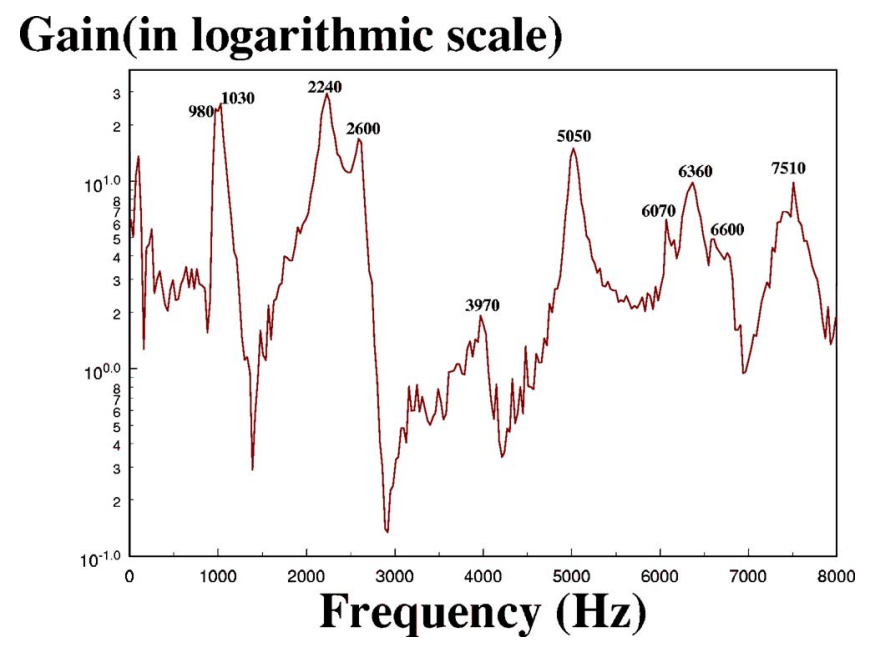

FIG. 9. Frequency response of the piezolaminated composite plate obtained by LDV-DSA system. 
TABLE VII. Results of resonant frequencies for the in-plane vibration obtained from AF-ESPI, impedance and FEM for the piezolaminated composite plate. (Error is compared with AF-ESPI values.)

\begin{tabular}{cccccc}
\hline \hline Mode & AF-ESPI (Hz) & Impedance (Error \%) & FEM I (Error \%) & FEM II (Error \%) & FEM III (Error \%) \\
\hline 1 & 22975 & $23089(0.50)$ & $22924(-0.22)$ & $22713(-1.14)$ & $23660(2.98)$ \\
2 & 53450 & $53573(0.23)$ & $53162(-0.54)$ & $51553(-3.55)$ & $52562(-1.66)$ \\
3 & 59400 & $59570(0.29)$ & $60331(1.57)$ & $58745(-1.10)$ & $58842(-0.94)$ \\
4 & 66240 & $66567(0.49)$ & $65767(-0.71)$ & $64426(-2.74)$ & $68611(3.58)$ \\
5 & 72800 & $73064(0.36)$ & $72745(-0.08)$ & $70652(-2.95)$ & $73075(0.38)$ \\
6 & 74060 & $74063(0.00)$ & $74563(0.68)$ & $72920(-1.54)$ & $74353(0.40)$ \\
\hline \hline
\end{tabular}

and FEM II (FEM III) is slightly larger than that between experimental measurement and FEM I. The material constants of the composite laminate used for FEM II (FEM III) are only nominal, therefore there might be differences between them and actual constants used in the experiments. Compare the results obtained by FEM II and FEM III, it is interesting to note that most of the results for resonant frequencies predicted by the FEM III are larger than those by FEM II. It is found that the piezoelectric effect only leads to a slighter increase in the rigidity of piezolaminated plates. The influence of the electric field on the free vibrations of piezoelectric materials was also investigated by Krommer and Irschik $^{18}$ and Borrelli and Patria. ${ }^{19}$

\section{CONCLUSIONS}

It is known that the resonant characteristics of piezoceramic plates are important in many engineering applications. However, there are only very few experimental results available in the literature. This study investigates the resonant frequencies and mode shapes of piezolaminated composite plate for out-of-plane and in-plane vibrations by three experimental techniques (AF-ESPI, LDV, impedance) and FEM. It has been shown that the AF-ESPI method has the advantages of noncontact, full-field, real-time, and highresolution measurement. Excellent quality of the interferometric fringe for the mode shape is presented by a video recording system. For the thin piezoceramic plate, the resonant frequencies and full-field mode shapes for up to 26 modes are presented by AF-ESPI and are excellently correlated with FEM results. It is found that the soft sponge support has a significant influence on the resonant frequencies of the lower resonant modes for thin piezoceramic plates. The finite element simulation underestimates the resonant frequencies for thin piezoceramic plates if the effect of the sponge support is not included. For the case of the piezolaminated composite plate, not only transverse modes but also in-plane modes are analyzed by three experimental techniques and FEM. Excellent agreement between the theoretical predictions and experimental measurements of resonant frequencies and mode shapes is obtained. The electric boundary conditions applied on the piezoceramic plate of the piezolaminated composite specimen have nearly no influence on the resonant frequencies for the out-of-plane modes, but significant influences are found for the in-plane vibration modes. The results indicated in this study also demonstrate that the experimental techniques proposed herein are applicable in the resonant analysis for piezolaminated plates.

\section{ACKNOWLEDGMENTS}

The authors gratefully acknowledge the financial support of this research by the National Science Council (Republic of China) under Grant No. NSC 90-2212-E-231-003.

${ }^{1}$ S. K. Ha, C. Keilers, and F. K. Chang, "Finite element analysis of composite structures containing distributed piezoceramic sensors and actuators," AIAA J. 30, 772-780 (1992).

${ }^{2}$ R. C. Batra and X. Q. Liang, "Vibration of a rectangular laminated elastic plate with embedded piezoelectric sensors and actuators," Comput. Struct. 63, 203-216 (1997).

${ }^{3} \mathrm{H}$. Abramovich and H. R. Meyer-Piening, "Induced vibrations of piezolaminated elastic beams," Compos. Struct. 43, 47-55 (1998).

${ }^{4}$ M. A. R. Loja, J. I. Barbosa, C. M. Mota Soares, and C. A. Mota Soares, "Analysis of piezolaminated plates by the spline finite strip method," Comput. Struct. 79, 2321-2333 (2001).

${ }^{5}$ J. N. Butters and J. A. Leendertz, "Speckle patterns and holographic techniques in engineering metrology," Opt. Laser Technol. 3, 26-30 (1971).

${ }^{6} \mathrm{R}$. Jones and C. Wykes, Holographic and Speckle Interferometry (Cambridge University Press, New York, 1982).

${ }^{7} \mathrm{~K}$. Hogmoen and O. J. Løkberg, "Use of modulated reference wave in electronic speckle pattern interferometry,” J. Phys. E 9, 847-851 (1976). ${ }^{8}$ S. Nakadate, "Vibration measurement using phase-shifting speckle pattern interferometry," Appl. Opt. 25, 4162-4167 (1986).

9 J. D. Valera, A. F. Doval, and J. D. C. Jones, "Determination of vibration phase with electronic speckle pattern interferometry (ESPI)," Electron. Lett. 28, 2292-2294 (1992).

${ }^{10} \mathrm{~W}$. O. Wong, K. T. Chan, and T. P. Leung, "Contrast and sensitivity of the vibration fringes in time-averaged electronic speckle-pattern interferometry: Effect of variations of force level," Opt. Laser Technol. 29, 179-185 (1997).

${ }^{11}$ W. C. Wang, C. H. Huang, and S. Y. Lin, "Vibration measurement by the time-averaging electronic speckle pattern interferometry method," Appl. Opt. 35, 4502-4509 (1996).

${ }^{12}$ W. C. Wang and Y. H. Tsai, "Experimental vibration analysis of the shadow mask," Opt. Lasers Eng. 30, 539-550 (1998).

${ }^{13} \mathrm{C}$. C. Ma and C. H. Huang, "The investigation of three-dimensional vibration for piezoelectric rectangular parallelepipeds using the AF-ESPI method," IEEE Trans. Ultrason. Ferroelectr. Freq. Control 48, 142-153 (2001).

${ }^{14} \mathrm{C}$. H. Huang and C. C. Ma, "Vibration characteristics for piezoelectric cylinders using amplitude-fluctuation electronic speckle pattern interferometry," AIAA J. 36, 2262-2268 (1998).

${ }^{15}$ ABAQUS User's Manual, ver. 6.2, Hibbit, Karlsson, and Sorensen, Inc, Pawtucket, RI, 2001.

${ }^{16}$ H. Y. Lin, J. H. Huang, and C. C. Ma, "Vibration analysis of angle-ply laminated composite plates with an embedded piezoceramic layer," IEEE Trans. Ultrason. Ferroelectr. Freq. Control 50, 1084-1099 (2003).

${ }^{17}$ C. K. Lee and G. Y. Wu, "High performance Doppler interferometer for advanced optical storage system," Jpn. J. Appl. Phys., Part 1 38, 17301741 (1999)

${ }^{18} \mathrm{M}$. Krommer and H. Irschik, "On the influence of the electric field on free transverse vibrations of smart beams," Smart Mater. Struct. 8, 401-410 (1999).

${ }^{19}$ A. Borrelli and M. C. Patria, "Saint-Venant's principle for a piezoelectric body," SIAM J. Appl. Math. 59, 1098-1111 (1999). 
Copyright of Journal of the Acoustical Society of America is the property of American Institute of Physics and its content may not be copied or emailed to multiple sites or posted to a listserv without the copyright holder's express written permission. However, users may print, download, or email articles for individual use. 